ORIGINAL ARTICLE

\title{
Bread consumption patterns in a Swedish national dietary survey focusing particularly on whole-grain and rye bread
}

\author{
Pernilla Sandvik'*, Iwona Kihlberg', Anna Karin Lindroos², Ingela Marklinder' and \\ Margaretha Nydahl' \\ 'Department of Food, Nutrition, and Dietetics, Uppsala University, Uppsala, Sweden; ${ }^{2}$ Swedish National Food Agency, \\ Uppsala, Sweden
}

Abstract

Background: Bread types with high contents of whole grains and rye are associated with beneficial health effects. Consumer characteristics of different bread consumption patterns are however not well known.

Objective: To compare bread consumption patterns among Swedish adults in relation to selected sociodemographic, geographic, and lifestyle-related factors. For selected consumer groups, the further aim is to investigate the intake of whole grains and the context of bread consumption, that is, where and when it is consumed.

Design: Secondary analysis was performed on bread consumption data from a national dietary survey $(n=1,435)$. Respondents were segmented into consumer groups according to the type and amount of bread consumed. Multiple logistic regressions were performed to study how selected socio-demographic, geographic, and lifestyle-related factors were associated with the consumer groups. Selected consumption groups were compared in terms of whole-grain intake and consumption context. Consumption in different age groups was analysed more in detail.

Results: One-third of the respondents consumed mainly white bread. Socio-demographic, geographic, and healthy-lifestyle-related factors were associated with the bread type consumed. White bread consumption was associated with younger age groups, less education, children in the family, eating less fruit and vegetables, and more candy and snacks; the opposite was seen for mainly whole-grain bread consumers. Older age groups more often reported eating dry crisp bread, whole-grain bread, and whole-grain rye bread with sourdough whereas younger respondents reported eating bread outside the home, something that also mainly white bread eaters did. Low consumers of bread also consumed less whole grain in total.

Conclusions: Traditional bread consumption structures were observed, as was a transition among young consumers who more often consumed fast food bread and bread outside the home, as well as less rye and whole-grain bread. Target groups for communication strategies and product development of more sensorily attractive rye or whole-grain-rich bread should be younger age groups (18-30 years), families with children, and groups with lower educational levels.

Keywords: public health; whole-grain bread; consumption context

Responsible Editor: Anja Olsen, Danish Cancer Association, Denmark.

Received: I0 February 20 I4; Revised: 25 May 20।4; Accepted: II August 20 I4; Published: 5 September 2014

$\mathrm{B}$ read is the primary source of carbohydrates in most parts of Europe (1) and has also been an important source of whole grain in the Scandinavian countries (2). From a health perspective, it is important to differentiate between different types of bread because the content can vary widely, from whole-grain rye bread with whole kernels and sourdough to white wheat bread with sifted flour, that is, not whole grain. However, according to a review by Frølich, Åman and Tetens (3) most of the wheat and rye consumed in Scandinavia today is in the form of sifted flour. From a public health perspective, in Sweden, as well as in other countries, there is a need to increase the consumption of whole grain $(2,4)$, especially among young adults $(5,6)$. Whole-grain intake is related to several health benefits such as a reduced risk of coronary heart disease, insulin resistance and type 2 diabetes, undesired weight gain, and colorectal cancer (7-9).

In Sweden, the National Food Agency has suggested five specific dietary recommendations for healthy eating, one of which is to choose primarily whole-grain products 
when eating cereal food (10). Here whole grain is defined as the whole-grain kernel (endosperm, germ, and bran); the kernel may be ground, crushed or similar but the components should be included in their original proportion for respective grain (10). Grains refer to wheat, including spelt, rye, oats, barley, maize, rice, millet, sorghum, and other sorghum varieties. From the perspective of carbohydrate quality, it is also important to differentiate between types of cereal included in the bread. Both endosperm and whole-grain rye, for example, have shown insulin-saving effects compared to wheat $(11,12)$ and have also been shown to be more satiating $(13,14)$. There has been much debate in Swedish media since the early 2000s regarding health benefits related to a lowered consumption of carbohydrates (15). Gunnarsson and Elam (16) discuss how the 'low-carb-high-fat' movement achieved a level of public credence and credibility while continuing to lack an established scientific basis for its dietary advice. It is not clear whether this may have affected the consumption of bread, but a longitudinal intervention program in the north of Sweden shows a trend break involving decreased reported intake of carbohydrates and an increased intake of fat beginning in 2004 (17). However, in the most recent Swedish national dietary survey Riksmaten 2010-11, 98\% reported eating bread (5). The total consumption was $75 \mathrm{~g} /$ day (SD 41) for women and $102 \mathrm{~g} /$ day (SD 55) for men and does not seem to have changed compared with earlier national dietary studies (from 1989 to 1997-98). In the national dietary survey from 1997-98, all participants reported that they had eaten bread, and the most commonly reported bread was classified as 'white bread' (18). In Riksmaten 2010-11, however, 'medium coarse bread' with 5-9\% dietary fibre was the most commonly reported (5).

The Swedish National Food Agency has only presented overall results on bread consumption from the dietary survey Riksmaten 2010-11 which includes mean total intake of bread types classified as: dry crisp bread, $9 \mathrm{~g} /$ day (SD 12); white wheat bread, $24 \mathrm{~g}$ (SD 31); sifted rye bread, $7 \mathrm{~g}$ (SD 20); medium coarse bread with 5-9\% fibre, $44 \mathrm{~g}$ (SD 39); and extra coarse bread, $3 \mathrm{~g}$ (SD 12). Bread contributed with on average $11 \%$ of the total energy intake and $51 \%$ of the whole-grain intake. Total intake for sex and age groups was also reported. This differed significantly between age groups of men but not women. The youngest (18-30 years) and oldest (65-80 years) age groups reported significantly less bread ( 90 and $93 \mathrm{~g} /$ day, respectively) compared to the age groups 30-44 years (112 g/day) and 45-64 years (106 g/day) (5).

Secondary analysis of dietary survey data provides a more in-depth understanding of both the consumption and consumer patterns for a specific food group. This is important information for forming strategies to favour the intake of bread rich in whole grain or rye - for example, in the targeting of both dietetic communication and product development. The analysis can be used to describe the characteristics of different consumer groups based on the type of bread reported, for example, in relation to socio-demographic, geographic, and life-style related factors. It also provides a more detailed picture of the eating context, where and when the food is consumed, and the significance of bread in the diet as a whole-grain source. To our knowledge, studies regarding consumers of different types of bread, the total intake, and the context of bread consumption have not previously been performed in a national representative setting in Sweden. Studies from Finland, however, have shown that a higher intake of rye bread was associated with lower occupational social class (19). Both white and rye bread consumption were associated with a lower educational level. Neither white nor rye bread was associated with a healthier lifestyle, but white bread was associated with an unhealthier lifestyle (20). In Australia, white bread has been shown to be eaten more often by consumers with less education, but also by younger consumers, and consumers with children (21). Cultural differences have also been related to cereal intake, as it was shown that among the Scandinavian countries in the 1990s, rye contributed most to the whole-grain intake in Denmark about $70 \%$; in Sweden - about $50 \%$; and in Norway about 20\% (2). According to statistics from the Swedish Board of Agriculture on the total supply of foods to individual households and catering, the consumption of flour decreased from 20 to $10 \mathrm{~kg}$ per person between the years 1960 and 2012. Instead the intake of industrially produced bread increased from 31 to $50 \mathrm{~kg}$ per person and year but the intake of dry crisp bread decreased from 7 to $3 \mathrm{~kg}$ (22). There has traditionally been local variation in Swedish bread culture, something that was still evident in the 1990s when more dry crisp bread was consumed in the north compared to the south (23). The world is becoming increasingly globalised and we receive culinary influences from different parts of the world. This may affect our eating pattern in relation to bread, which might also have consequences from a nutritional perspective.

The aim of this study is to compare bread consumption patterns among Swedish adults in relation to selected socio-demographic, geographic, and lifestyle-related factors. For selected consumer groups, the further aim is to investigate the intake of whole-grain and rye bread and the context of bread consumption, that is, where and when bread is consumed.

\section{Material and method}

In a national dietary survey, Riksmaten 2010-11, dietary data from Swedish adults (18-80 years) was collected (5). In the present study secondary analyses were performed to provide more detailed insight into the consumption of 
bread. The dietary survey consisted of a 4-day estimated food diary and a questionnaire with about 50 separate questions. The food record was computer-based and self-administrated. Bread was registered in pieces or slices by respondents in an online database. The database (24) had a selection of 1,900 food items, including 62 different bread types representing breads available on the Swedish market, with nutritional values including whole-grain content specified. Place of consumption was also reported - for example, at home, in a restaurant, etc. Separate questions relevant to the present study were: number of children $<18$ years in the household, physical activity level, educational level, country of birth, and some specific food frequency questions (FFQ). Information on geographical region, sex, and age was obtained from the population database with the objective of including a representative national sample. For participants who did not have access to the Internet or did not want to report their intake online, it was possible to report dietary intake by telephone. The study was approved by the Central Ethical Review Board in Uppsala, Sweden (Ref. 2010/060).

The present analysis includes 1,435 respondents (response rate: $36 \%$ ). The main sample of the survey included 4,000 adults selected from the population registry. The population was stratified by sex, age group (18-30, 31-44, 45-64, and 65-80), and region. From each stratum, a random sample was drawn four times over the data collection period (May 2010-June 2011). Statistics Sweden carried out the sample selection and contacted participants, by telephone, postal invitation letters and reminders. The most commonly reported reasons for not participating were lack of interest and lack of time. About one-fifth of the invited sample could not be reached by telephone.

\section{Classification of breads and consumers}

To study differences between those with lower and higher total consumption of bread, respondents were stratified by median total intake in dry weight $(69 \mathrm{~g})$. In the classification, dry weight of all bread types was used to adjust for the lower water content in dry crisp bread, and intake was adjusted for total energy intake (per $10 \mathrm{MJ}$ ).

Segmentation of respondents into bread type groups was based on relative intake of four bread categories defined by the present research group. These categories were somewhat different from the bread categories used in the Riksmaten report (5) to enable a classification of the respondents. One was dry crisp bread, a traditional bread type in the Nordic countries that is often made from whole-grain flour, especially rye. The other three were based on the total content of whole grain. Bread with whole grain was classified into two groups due to the range of bread, including only a low content of whole grain on the Swedish market.
1. Dry crisp bread: Traditional Swedish bread with low water content often made from whole-grain rye and sometimes sifted flour.

2. White bread: With no whole grain, for example, white wheat roll, baguette, and sifted rye bread such as traditional Swedish wheat-rye loaf.

3. Bread with $<\mathbf{2 5 \%}$ whole grain: Medium coarse bread types including whole grain but $<25 \%$, for example, loafs, rolls.

4. Whole-grain bread $\geq \mathbf{2 5} \%$ whole grain (whole-grain bread): Coarser bread also including, for example, pumpernickel bread types with whole kernels.

The respondents were classified into main consumers of the respective bread types by using the proportional reported intake relative to the total amount of bread reported by each respondent. The theoretical value of this variable was thus $0-1$. A respondent may for example have reported on average $120 \mathrm{~g}$ bread per day with a proportion of 0.67 white bread, 0.08 bread with $<25 \%$ whole grain, 0.16 whole-grain bread, and 0.08 dry crisp bread. This respondent would be classified as a white bread consumer. The respondent was classified into the bread category that he or she had reported relatively most of. Since respondents consuming mainly dry crisp bread and bread with $<25 \%$ whole grain were few, all respondents in the fourth quartile of relative intake were included to ensure that the groups were large enough for analysis (resulting in a small overlap of respondents). In the classification for white bread and whole-grain bread consumers (which mainly are compared) the groups are non-overlapping. Non-bread consumers $(n=26)$ and ties $(n=23)$ were excluded here.

For further insight into the different consumption patterns between sex and age groups (18-30 years, 31-44 years, $45-64$ years, and $65-80$ years) a more detailed classification with 23 groups of bread was used. The percentage of respondents who reported eating a bread type was calculated for each group and these were compiled in a twoway contingency table. Because of the special interest in rye in the present study, the amount of sifted rye bread reported was also calculated for respondents with and without children ( $<18$ years) living at home.

\section{Socio-demographic, geographical, and healthy-lifestyle- related consumer characteristics}

Indicators of socio-demography used to compare the consumer groups were sex, age groups, having children $<18$ years living at home, number of years in school ( $<9$ years, $10-13$ years, or $\geq 14$ years), and country of birth (Sweden, other Nordic country, other parts of Europe, or countries outside Europe). Living in the south (Götaland), middle (Svealand), or north (Norrland) of Sweden was used to represent geographical differences. Factors representing a healthier lifestyle were self-reported 
physical leisure-time activity level (sedentary; moderate, 1-2 times per week; or 3 or more times per week), fruit and vegetable intake (below/above median reported by FFQ questions), and candy and snack intake (above/below median for candy and chocolate, cakes, crisps, and soda intake reported by the FFQ).

\section{Intake of whole grain and consumption context}

Low versus high consumers of bread in total and the most different consumer groups regarding intake of wholegrain bread, white bread versus whole-grain bread consumers, were further compared regarding total intake of whole grain. These groups were also compared regarding consumption context, that is, reported total intake of bread at different locations (home, restaurant/on the run). Whole-grain intake from bread was also calculated for different age groups and percentage of respondents in age/sex groups reporting bread at different locations and for different type of meals were also analysed.

\section{Statistical analysis}

To study the association between the consumption groups and selected socio-demographic, geographical, and lifestyle-related factors, five multiple logistic regression models were calculated (25). Binary dependent variables for type of bread were ' 1 ' for primarily consuming this bread, 0 ' for not, and for total consumption; ' 1 ' for higher consumers of total bread, and ' 0 ' for lower consumers of total bread. The associations are presented in odds ratios (OR) with 95\% confidence intervals (95\% CI). The Hosmer and Lemeshow goodness-of-fit test (26) confirmed the appropriateness of the models.

Independent sample $t$-tests were used for comparing total intake of whole grain among low versus high bread consumers, whereas main consumers of white bread versus main consumers of whole-grain bread and intake of bread at different locations were compared using a Mann-Whitney U test in these groups. Differences in consumption context among males and females in different age groups were calculated using chi-square based on percentage of respondents. The difference in the mean intake of sifted rye bread was calculated using the Mann-Whitney U test. To give a graphical illustration of the contingency table crossing the frequency of reporting different bread types among sex and age groups, Correspondence analysis was used. This is a descriptive/ exploratory technique designed to analyse two-way contingency tables (25). All statistical analyses were performed in the Statistical Package for the Social Sciences, version 19 (27).

\section{Results}

The distribution of respondents as to sex, age group, education, and birth country compared to overall national data from Statistics Sweden (2010) are shown in Table 1.
Table 1. Total respondents $(\%)(n=1,435)$ compared to the Swedish population in general

\begin{tabular}{lcc}
\hline & Respondents \% $(n)$ & Swedish population \%* \\
\hline Female & $56(807)$ & 50 \\
Male & $44(628)$ & 50 \\
Age group & & \\
$\quad 18-30$ & $19(270)$ & 23 \\
$31-44$ & $23(336)$ & 25 \\
$45-64$ & $38(542)$ & 34 \\
$65-80$ & $20(287)$ & 18 \\
School years & & \\
$\leq 9$ & $14(195)$ & 21 \\
$10-14$ & $58(835)$ & 60 \\
$>14$ & $28(405)$ & 19 \\
Born outside Sweden & $9(136)$ & 17 \\
\hline
\end{tabular}

*Statistics Sweden 2010.

Approximately one- third (36\%) of the respondents were classified as mainly white bread consumers, and $37 \%$ were classified as mainly whole-grain bread consumers. The mean reported daily bread intake was 56 g/day (SD 31) for low and $115 \mathrm{~g} /$ day (SD 45) for high consumers of bread in total. The reported intake of the bread types for main consumers of white bread, bread with $<25 \%$ whole grain, whole-grain bread, and dry crisp bread are shown in Table 2.

Socio-demographic, geographical, and healthy-lifestyle factors in relation to consumption groups

The computed ORs and CIs for being classified as a main consumer of the different bread types (white bread, bread with $<25 \%$ whole grain, whole-grain bread, and dry crisp bread) and for being a high versus a low consumer of bread in total are shown in Table 3.

There was a higher likelihood of being a mainly wholegrain bread consumer among the older age groups (45-64 years and 65-80 years) compared to the younger, whereas the opposite was seen for eating mainly white bread. Older respondents also had a higher likelihood of eating more bread in total. In terms of sex, the only significant effect was seen for dry crisp bread, with males having a lower likelihood of eating mainly this bread type (OR: 0.74; CI: 0.56-0.99). Although not significant, the tendency of the ORs also shows a higher likelihood among men of being mainly white bread consumers and eating more bread in total. Respondents with children had a higher likelihood of being mainly white bread consumers (OR: 1.42; CI: 1.08-1.96) and also a lowered likelihood of eating mainly dry crisp bread (OR: 0.61; CI: 0.43-0.87). On average 19\% of the reported white bread could be classified as bread that also included some amount of sifted rye. Respondents with children $<18$ years living at home reported significantly ( $p=0.005)$ more of this bread type ( $9 \mathrm{~g} /$ day SD 24$)$ compared to other respondents (7 g/day SD 19). 
Table 2. Mean reported intake of bread (g/day) among low and high bread consumers and main consumers of white bread, bread with $<25 \%$ whole grain, whole-grain bread and dry crisp bread

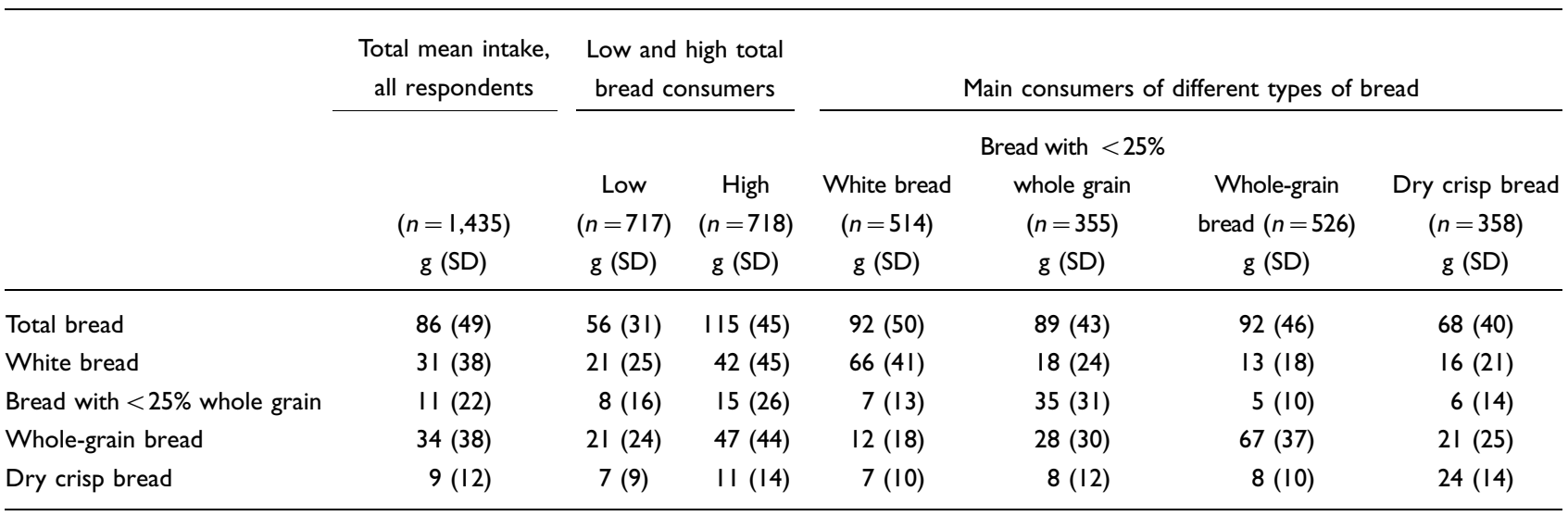

SD: standard deviation.

Country of birth was associated with eating mainly whole-grain bread with a higher likelihood for those born in a Nordic country other than Sweden (OR: 2.23; CI: $1.12-4.45)$. This was however a small group of respondents, which is reflected by the wider confidence intervals. The trend for number of years in school is a lowered likelihood of being mainly a white bread consumer with more school years. The opposite was seen for mainly whole-grain bread consumers and more years in school also lowered the likelihood of being a high bread consumer compared to a low.

According to geographical difference, region in Sweden showed to be the most important factor for dry crisp bread, with a higher likelihood in the north of Sweden (OR 3.24; CI: 2.17-4.83). Although dry crisp bread most often is made of whole-grain rye, white 'thin' crisp bread mostly made of wheat is also traditionally consumed more of in the north of Sweden. This was also seen in the present study where it contributed with $7 \%$ of the total dry crisp bread consumption in comparison to $4 \%$ in the general population. Even so, the consumption of dry crisp bread is higher in the north of Sweden also when excluding this white 'thin' crisp bread.

Among the health-related lifestyle factors, a more frequent candy and snacks intake was associated with being a white bread consumer (OR: 1.69; CI: 1.30-2.20) and the opposite was seen for mainly whole-grain bread consumers (OR: 0.62; CI: 0.47-0.81). A more frequent intake of fruit and vegetables was associated with a lowered risk of eating mainly white bread and vice versa for whole-grain bread and dry crisp bread. The trend was similar although not significant for a higher level of physical leisure activity. A higher total bread intake was also associated with a high level of physical leisure activity (OR 1.59; CI 1.02-2.49) whereas a high candy and snacks intake showed a lowered OR (0.77; CI: 0.69-0.98).
Total whole-grain intake among different consumer groups and whole-grain intake from bread according to sex and age groups

For total intake of whole grain there were significant differences when comparing low versus high total bread consumers and mainly white bread versus mainly whole-grain bread consumers. Mainly white bread consumers had a mean total intake of $38 \mathrm{~g} /$ day (SD 31) and mainly whole-grain bread consumers reported $45 \mathrm{~g} /$ day (SD 31) $(p \leq 0.001)$. The reported total intake of whole grain was $38 \mathrm{~g} /$ day (SD 29) for low bread consumers, compared to $45 \mathrm{~g} /$ day (SD 33 ) for high bread consumers $(p \leq 0.001)$. Intake of whole grain from bread also significantly differed between age groups: 18-30 years, average $14 \mathrm{~g}$ whole grain/day (SD 15) from bread; 31-44 years, $17 \mathrm{~g} /$ day (SD 15), 45-64 years, 22 g/day (SD 17), and the oldest age group, 65-80 years, $25 \mathrm{~g} /$ day (SD 16).

\section{Correspondence analysis of registered bread types among sex and age groups}

The first dimension in the correspondence analysis (Fig. 1) distinguishes between the younger (18-30 years and 31-44 years) and older (45-64 years and 65-80 years) age groups. To the right, older respondents are found together with dry crisp bread, whole-grain bread, and whole-grain rye bread. To the left are younger respondents together with, for example, tortilla, hamburger rolls, and pita bread. The two younger and older age groups are more alike for women than for men. Dimension 2 distinguishes between the sexes. At the bottom we find men and bread types categorised as loaves or toast, sliced bread. Men in the age group 31-44 years are situated close to pita bread, sausage roll and loaf with wheat, sifted rye and syrup.

\section{Context of bread consumption according to place and type of meal}

On average, most of the bread $(62 \%)$ was eaten at home, about $12 \%$ at work/lunch room, about $8 \%$ at a restaurant, 
Table 3. The odds for respondents with different socio-demographic, geographic, and lifestyle-related characteristics of being classified as main consumer of different types of bread and being a high versus a low consumer of total bread

\begin{tabular}{|c|c|c|c|c|c|}
\hline $\begin{array}{l}\text { Independent } \\
\text { Factors }(n)^{*}\end{array}$ & $\begin{array}{l}\text { Main consumers, } \\
\text { white bread } \\
\text { OR }(95 \% \mathrm{Cl})\end{array}$ & $\begin{array}{c}\text { Main consumers, } \\
\text { bread }<25 \% \text { whole grain } \\
\text { OR }(95 \% \mathrm{Cl})\end{array}$ & $\begin{array}{l}\text { Main consumers, } \\
\text { whole-grain bread } \\
\text { OR }(95 \% \mathrm{Cl})\end{array}$ & $\begin{array}{l}\text { Main consumers, } \\
\text { Dry crisp bread } \\
\text { OR }(95 \% \mathrm{Cl})\end{array}$ & $\begin{array}{l}\text { High bread } \\
\text { consumers } \\
\text { OR }(95 \% \mathrm{Cl})\end{array}$ \\
\hline \multicolumn{6}{|l|}{ Sex } \\
\hline Female (694) & 1.00 & 1.00 & 1.00 & 1.00 & 1.00 \\
\hline Male $(5 \mid 4)$ & $1.20(0.92-1.56)$ & $0.95(0.72-1.26)$ & $0.91(0.70-1.18)$ & $0.74(0.56-0.99)^{a}$ & $\mathrm{I} .89(0.97-1.57)$ \\
\hline \multicolumn{6}{|l|}{ Age } \\
\hline $18-30(220)$ & 1.00 & 1.00 & 1.00 & 1.00 & 1.00 \\
\hline $3 \mathrm{I}-44(286)$ & $0.76(0.5 \mathrm{I}-1.13)$ & $1.22(0.78-1.91)$ & $1.12(0.80-1.87)$ & $1.35(0.80-2.26)$ & I.4I (0.97-2.06) \\
\hline $45-64(465)$ & $0.57(0.40-0.8 \mathrm{I})^{\mathrm{b}}$ & I.II (0.42-I.67) & $1.63(1.12-2.36)^{a}$ & $2.05(1.33-3.18)^{b}$ & $1.42(1.01-2.49)^{\mathrm{a}}$ \\
\hline $65-80(237)$ & $0.33(0.2 I-0.5 I)^{c}$ & $1.69(1.08-2.66)^{\mathrm{a}}$ & $1.58(1.04-2.41)^{\mathrm{a}}$ & $3.15(1.96-5.01)^{c}$ & $1.68(1.03-1.48)^{b}$ \\
\hline \multicolumn{6}{|c|}{ Children $<18$ living at home } \\
\hline $0(776)$ & 1.00 & 1.00 & 1.00 & 1.00 & 1.00 \\
\hline$\geq I(432)$ & $1.42(1.08-1.96)^{\mathrm{b}}$ & $1.20(0.87-1.66)$ & $0.79(0.58-1.06)$ & $0.61(0.43-0.87)^{b}$ & $1.12(0.85-1.48)$ \\
\hline \multicolumn{6}{|l|}{ Birth country } \\
\hline Sweden (I I02) & 1.00 & 1.00 & 1.00 & 1.00 & 1.00 \\
\hline Nordic excl. Swe.(38) & $0.7 \mathrm{I}(0.3 \mathrm{I}-\mathrm{I} .6 \mathrm{I})$ & $0.88(42-1.85)$ & $2.23(1.12-4.45)^{\mathrm{a}}$ & $0.54(0.25-1.18)$ & $0.85(0.44-1.62)$ \\
\hline Europe (36) & $0.94(0.44-2.01)$ & $0.79(0.35-1.79)$ & $1.07(0.54-2.14)$ & $1.43(0.67-3.08)$ & $1.10(0.56-2.17)$ \\
\hline Outside Europe (32) & $1.89(0.88-4.07)$ & $0.94(0.40-1.24)$ & $0.44(0.18-1.11)$ & $\mathrm{I} .40(0.59-3.3 \mathrm{I})$ & $0.55(0.27-1.14)$ \\
\hline \multicolumn{6}{|l|}{ School years } \\
\hline$\leq 9$ yrs. I54) & 1.00 & 1.00 & 1.00 & 1.00 & 1.00 \\
\hline $10-14$ years $(694)$ & $0.59(0.40-0.86)^{b}$ & $0.84(0.56-1.26)$ & $1.35(0.91-2.00)$ & $0.94(0.62-1.14)$ & $0.82(0.57-1.17)$ \\
\hline$>14$ yrs. $(360)$ & $0.50(0.33-0.77)^{b}$ & $0.85(0.54-1.32)$ & $\mathrm{I} .44(0.93-2.2 \mathrm{I})$ & $0.99(0.63-1.56)$ & $0.64(0.44-0.95)^{\mathrm{a}}$ \\
\hline \multicolumn{6}{|l|}{ Region in Sweden } \\
\hline South (583) & 1.00 & 1.00 & 1.00 & 1.00 & 1.00 \\
\hline Middle (47I) & $1.10(0.84-1.45)$ & $0.95(0.72-1.26)$ & $0.87(0.69-1.12)$ & $1.72(1.23-2.31)^{c}$ & $0.88(0.69-1.13)$ \\
\hline North (154) & $1.45(0.98-2.11)$ & $0.70(0.46-1.09)$ & $0.62(0.4 I-0.92)^{a}$ & $3.24(2.17-4.83)^{c}$ & $0.78(0.55-1.17)$ \\
\hline \multicolumn{6}{|l|}{ Physical activity } \\
\hline Sedentary (106) & 1.00 & 1.00 & 1.00 & 1.00 & 1.00 \\
\hline Moderate (50I) & $0.85(0.54-1.32)$ & $1.24(0.74-2.06)$ & $1.09(0.68-1.75)$ & $0.73(0.44-1.21)$ & $1.54(1.01-2.35)^{\mathrm{a}}$ \\
\hline I-2 times/week (262) & $0.56(0.38-1.01)$ & $1.47(0.85-2.55)$ & $1.21(0.72-2.02)$ & $0.77(0.45-1.34)$ & $1.18(0.75-1.87)$ \\
\hline$\geq 3$ times/week (339) & $0.71(0.44-1.15)$ & $0.88(0.5 \mathrm{I}-1.53)$ & $1.49(0.9 I-\mid .8 I)$ & $0.79(0.47-1.34)$ & $1.59(1.02-2.49)^{\mathrm{a}}$ \\
\hline \multicolumn{6}{|l|}{ Candy/snacks intake } \\
\hline Low (778) & 1.00 & 1.00 & 1.00 & 1.00 & 1.00 \\
\hline High (430) & $1.69(1.30-2.20)^{c}$ & I.II (0.83-I.47) & $0.62(0.47-0.8 I)^{c}$ & $0.82(0.60-1.11)$ & $0.77(0.60-0.98)^{a}$ \\
\hline \multicolumn{6}{|l|}{ Fruit/vegetable intake } \\
\hline Low (563) & 1.00 & 1.00 & 1.00 & 1.00 & 1.00 \\
\hline High (645) & $0.57(0.44-0.75)^{c}$ & $1.19(0.90-1.59)$ & $1.39(1.07-1.8 I)^{b}$ & $1.41(1.05-1.90)^{\mathrm{a}}$ & $0.94(0.73-1.20)$ \\
\hline
\end{tabular}

OR: odds ratio; $\mathrm{Cl}$ : confidence interval.

Reference categories for each independent factor are shown in parentheses in first column. Bold values and superscript letters indicate odds ratios, within a column, for a characteristic being significantly different from reference category ${ }^{\mathrm{a}} p \leq 0.05,{ }^{\mathrm{b}} p \leq 0.0 \mathrm{I},{ }^{\mathrm{c}} p \leq 0.00 \mathrm{I}$. $*$ There may be small variations in the number of participants of each analysis due to missing values.

and $4 \%$ 'on the run', and at friends'/relatives' places, respectively. Almost half of the bread consumed is reported for breakfast $(42 \%)$ and approximately $20 \%$ is reported for lunch, dinner and snacks, respectively. Mainly white bread consumers ate significantly less bread at home (56 g/day, SD 46) compared to mainly wholegrain bread consumers (66 g/day, SD 43, $p \leq 0.001)$ as well as more bread at restaurants and 'on the run' (18 g/day, SD 35, compared to $9 \mathrm{~g} /$ day, SD 24, $p \leq 0.001$ ). For sex and age groups there is a difference in the frequency of respondents reporting eating bread at different locations. Significantly more respondents in the younger age groups reported eating bread at relatives'/ friends' places, at restaurants, and on the run (Table 4). 


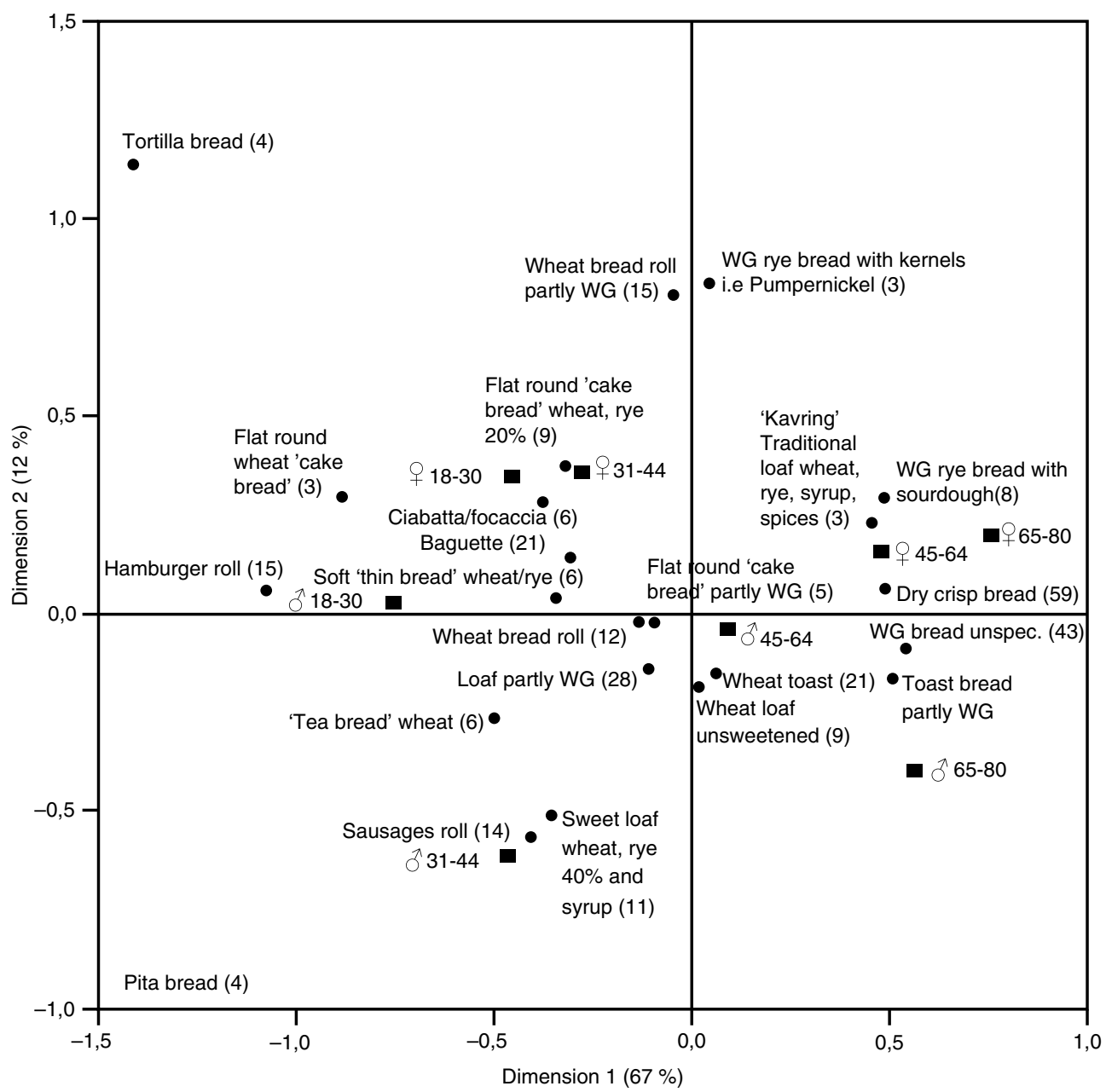

Fig. 1. Correspondence analysis of percentage of respondents that according to sex ( $q$ and $\widehat{\rho})$ and age groups reported eating different bread types. Numbers in brackets indicate total percentage of respondents reporting eating the specific bread type among a total of $n=1,435$. WG: whole grain.

A significantly lower number of the youngest respondents also reported eating bread at home. More women (58\%) than men $(51 \%)$ reported eating bread in between meals $(p=0.007)$ and more men $(25 \%)$ than women $(19 \%)$ at restaurants $(p=0.010)$.

\section{Discussion}

Findings from the present study show that one-third of the respondents mainly consumed bread without whole grain. Associated factors were lower educational level, children living at home, eating less fruit and vegetables, more candy and snacks, and younger age groups. A traditional eating pattern was observed with more dry crisp bread in the north part of Sweden and more whole-grain bread among respondents born in a Nordic country outside Sweden. Younger respondents reported to eat less bread in total, other types of bread, and in other eating contexts compared to older respondents, indicating a transition regarding bread consumption. Lower total bread consumption was related to a lower total intake of whole grain.
For type of bread, eating less fruit and vegetables and more candy and snacks was associated with a higher risk of eating mainly white bread and vice versa for the more whole-grain-rich bread types. Crisp bread, often made of whole-grain rye, was also associated with a higher fruit and vegetable intake. In western Sweden, white bread was earlier clustered together with a more unhealthy eating pattern and whole-grain bread with a healthier one (28). This was also seen for whole-grain intake in northern Sweden at the end of the 1990s (29). Regarding total consumption of bread, higher total bread consumption was more associated with higher physical activity level and lower intake of candy and snacks. Despite the recent media attention to proponents of low carbohydrate intake, a lower total intake of bread as a health strategy could not be seen in the present analysis.

A lower educational level was related to being a high consumer of bread in total compared to a low consumer as well as with a higher likelihood of eating mainly white bread. This is in line with earlier studies $(28,30)$ but 
Table 4. Percentage of respondents in sex and age groups reporting eating bread at different locations and meal types

\begin{tabular}{|c|c|c|c|c|c|c|c|c|c|c|}
\hline & \multicolumn{5}{|c|}{ Female } & \multicolumn{5}{|c|}{ Male } \\
\hline & $\begin{array}{c}18-30 \\
(n=161)\end{array}$ & $\begin{array}{c}31-44 \\
(n=196)\end{array}$ & $\begin{array}{c}45-64 \\
(n=292)\end{array}$ & $\begin{array}{c}65-80 \\
(n=158)\end{array}$ & $P$ & $\begin{array}{c}18-30 \\
(n=110)\end{array}$ & $\begin{array}{c}31-44 \\
(n=\mid 40)\end{array}$ & $\begin{array}{c}45-64 \\
(n=249)\end{array}$ & $\begin{array}{c}65-80 \\
(n=129)\end{array}$ & $P$ \\
\hline Home & $85^{\mathrm{a}}$ & $92^{\mathrm{b}}$ & $93^{\mathrm{b}}$ & $98^{\mathrm{b}}$ & $<0.001$ & $83^{a}$ & $94^{\mathrm{b}}$ & $94^{\mathrm{b}}$ & $99^{c}$ & $<0.001$ \\
\hline Work/lunch room & $36^{\mathrm{a}}$ & $43^{a, b}$ & $48^{\mathrm{b}}$ & $5^{c}$ & $<0.001$ & $29^{a}$ & $48^{\mathrm{b}}$ & $46^{\mathrm{b}}$ & $5^{c}$ & $<0.001$ \\
\hline Friends/relatives & $27^{\mathrm{a}}$ & $15^{\mathrm{b}}$ & $17^{b}$ & $15^{\mathrm{b}}$ & 0.013 & $21^{\mathrm{a}}$ & $14^{\mathrm{a}, \mathrm{b}}$ & $11^{\mathrm{b}}$ & $10^{\mathrm{b}}$ & 0.044 \\
\hline Restaurant & $31^{\mathrm{a}}$ & $19^{\mathrm{b}}$ & $16^{\mathrm{b}}$ & $11^{\mathrm{b}}$ & $<0.001$ & $23^{\mathrm{a}}$ & $36^{\mathrm{b}}$ & $25^{\mathrm{a}}$ & $12^{c}$ & $<0.001$ \\
\hline On the run & $19^{\mathrm{a}}$ & $14^{\mathrm{a}}$ & $8^{\mathrm{b}}$ & $I^{c}$ & $<0.001$ & $19^{a}$ & $18^{\mathrm{a}}$ & $12^{\mathrm{a}}$ & $5^{b}$ & 0.001 \\
\hline Event & 3 & 4 & 4 & 2 & 0.593 & 4 & 3 & 4 & 3 & 0.933 \\
\hline Breakfast & 82 & 81 & 81 & 84 & 0.888 & $76^{\mathrm{a}}$ & $91^{\mathrm{b}}$ & $85^{b}$ & $86^{\mathrm{a}, \mathrm{b}}$ & 0.011 \\
\hline Lunch & 85 & 84 & 86 & 82 & 0.607 & 88 & 84 & 87 & 89 & 0.664 \\
\hline Dinner & 65 & 65 & 64 & 67 & 0.953 & $54^{\mathrm{a}}$ & $68^{\mathrm{b}}$ & $68^{\mathrm{b}}$ & $70^{b}$ & 0.031 \\
\hline In between meals & 58 & 60 & 60 & 53 & 0.442 & 40 & 51 & 54 & 54 & 0.084 \\
\hline
\end{tabular}

$P<0.05$ indicate significant differences between age groups separately for females and males.

Each superscript letter denotes a subset of age categories for females and males separately whose column proportions do not differ significantly from each other at the 0.05 level.

indicates a difference in bread consumption compared with Finland, where whole-grain rye bread is related to lower occupational class (19). A higher chance of eating more bread in total can be related to bread being relatively cheap and not needing any preparation if it is commercially baked. The difference in consumption for bread types across educational levels may be related to price differences or a knowledge gap regarding the health effects of different types of bread, sensory aspects, or traditions. These are factors that have previously been suggested as barriers to whole-grain consumption $(31,32)$.

Despite geographical availability being less of a barrier today, traditional structures in relation to bread consumption still are evident. The difference between the south and north of Sweden (23) still remains, as dry crisp bread consumption is more associated with the north and soft whole-grain bread with the south. The analysis included few respondents born outside Sweden but it indicates a higher likelihood of consuming mainly wholegrain bread among respondents born outside Sweden but in another Nordic country. This finding probably reflects the fact that consumption of whole-grain rye bread has traditionally been higher in Finland and Denmark $(20,33)$. In relation to traditions and habits in different cultures one can speculate if there is today a sensory barrier to consumption of the most wholegrain-rich breads $(34,35)$. Cultural factors could be important to account for in attempts to enable a higher consumption of bread with health benefits.

Significant associations with age were found for all bread consumption groups. Dry crisp bread was strongly associated with older age groups. The youngest and the oldest age group of men consumed significantly less bread in the present dietary survey. The lower intake in the oldest age group is however probably due to a higher intake of crisp bread, which weighs less. Younger age groups (primarily 18-30 years) were associated with mainly white bread consumption, and earlier research has indicated differences in bread preference between age groups (36) which may be one explanation for these results. Having children in the household was also an associated factor regarding white bread consumption, which is in line with earlier studies $(21,28)$. In a family, food choices often are negotiated and conflicts may arise due to different values or preferences (37). The present result indicates the child's impact on the parent's bread consumption. One potential barrier to explore is if there is a difference in the convenience level for bread types, with, for example, more whole-grain rye compared to white bread such as package size and bread format (e.g., pre-sliced loaf or roll). Another potential reason why families with children eat less whole-grain bread may have to do with sensory aspects, that is, that taste has been shown to be a barrier among Swedish adolescents (11-15 years) to eating fibre-rich bread (38) although not among Finish adolescents (13-15 years) (39). What is eaten at younger ages might be important for future preferences, and Cooke (40) concludes, in a review of the importance of exposure to healthy eating in childhood, that 'experiences with flavours shape present and future preferences'. Bread including some amount of sifted rye was included in the white bread group and respondents with children consumed more of this type of bread. Although this bread in Sweden also often includes mostly sifted wheat, bread with at least $75 \%$ endosperm rye has been shown to have insulin-saving effects compared to white wheat bread (11). Besides increasing the whole-grain content, including more endosperm rye might be one way to improve the carbohydrate quality, while still maintaining the mild 
taste, because the intense flavour components of rye are situated mainly in the outer layers of the kernel (35).

Bread has traditionally filled an important part of the diet and it still does. The reported whole-grain intake in the present study (31-45 g/day among different bread consumers), however, does not reach the Swedish national dietary recommendations of consuming 70-90 g/day (10), not even among mainly whole-grain bread consumers. Even if they reported significantly more whole grain in total compared to white bread eaters, there is still a possibility of improving their bread consumption towards even more whole-grain-rich bread types. Important aspects to address could be the availability of tasty whole-grain-rich bread and how the choice of bread can be facilitated through, for example, labelling. Low bread consumers reported a significantly lower wholegrain intake than did consumers eating more bread in total. This indicates that bread remains a very important source of whole grain in the diet. Low consumers do not seem to sufficiently complement their lower intake with other whole-grain sources, despite the extended range of whole-grain products on the market (10). If less bread is eaten as a health strategy, this is an important issue to address.

One explanation for younger consumers eating more white bread might be a transition in how bread is consumed. The correspondence analysis (Fig. 1) indicates a difference in bread types eaten, with more influences from other cultures and bread types such as pita bread, tortilla, baguette, and hamburger rolls. Older age groups more often reported eating traditional bread types such as dry crisp bread, bread with whole grain, and wholegrain rye bread with sourdough. More respondents in the younger age groups have also reported eating bread outside the home, something that also mainly white bread eaters did. Meals outside the home contributed with less dietary fibre compared with meals at home, according to a previous European study (41), although this was most evident among the southern European countries. Eating bread outside the home might be one explanation for why younger age groups are associated with being mainly white bread consumers and have a lower intake of whole grain from bread. They also more often report eating bread types that may be found at fast food restaurants. Mere exposure is one factor related to the development of food preferences (42) and the lower consumption among younger consumers might therefore indicate a reduced acceptance of whole-grain rye bread in Sweden in the future.

It is well agreed that there are potential measurement errors in dietary assessment surveys that are important to bear in mind when interpreting the present results. Food records are reactive dietary assessment instruments that may alter the dietary behaviour of the subjects (43). Also important to bear in mind when analysing the present results is that there is no standardised bread classification in Sweden. The responders were asked to identify the bread in the database most similar to the bread they had consumed. Although the bread types listed aimed to reflect those on the Swedish market, there is a risk that bread was perceived differently. Classification of bread has also been challenging in an earlier study (44), where the categories ended up as 'white' or 'dark'. In the present analysis it was possible to make a rough classification of the included bread types and some more detailed analyses regarding specific bread types. Because, for example, the flour extraction rate per se does not alter the blood glucose response of bread (45) a future challenge is to investigate bread intake with particular reference to this aspect of bread quality. The response rate of this survey was low, which limits its representativeness. The response rate of the previous Swedish national food survey (Riksmaten 1997-98) was $60 \%$, considerably higher. This reflects the challenge of the downward trend that has been seen in several national survey studies in recent years $(46,47)$. A similarly low response rate $(37 \%)$ was observed in the most recent Norwegian national food survey (48). In line with previous studies $(49,50)$ the rate of non-responders was higher among lower educational levels and also among immigrants. Strengths of the present study were that detailed food records over 4 days were collected from all over Sweden and both sex and age groups were well represented in relation to the Swedish population (Table 1). The present study contributes with insights regarding bread consumption in Sweden in the year 2010 11 and in the present material traditional structures in bread consumption were still seen, but at the same time a pattern indicating a transition in eating was observed regarding both type of bread and eating context. Young respondents consumed less bread in total as well as less whole-grain bread, whole-grain rye bread, and dry crisp bread. This is a negative result from a public health perspective, according to the health aspects related to both whole grain and rye $(7-9,11-14)$. This may also be one reason for the lower whole-grain intake among younger consumers. A possible strategy to change this trend could be to improve the availability of more sensorily appealing whole-grain-rich bread at restaurants and breads to be consumed 'on the run'. From a methodological perspective, this way of analysing could also be performed among other food groups to identify potential avenues to promote healthier eating.

\section{Conclusion}

In the present national dietary survey, more than one-third of the respondents reported eating mainly white bread. Socio-demographic, geographic, and lifestyle-related factors are associated with what type of bread is consumed. Older age groups more often reported eating traditional 
bread types such as dry crisp bread, whole-grain bread, and whole-grain rye bread with sourdough. More respondents in the younger age groups reported eating bread outside the home, something that also mainly white bread eaters did. Development of more sensorily attractive rye or whole-grain-rich bread and eating-out types of bread is thus a challenge and should be in focus in further studies. Target groups for health communication strategies and product development should be younger age groups (18-30 years), families with children, and groups with lower educational levels.

\section{Conflict of interest and funding}

This publication is funded by the foundation Stiftelsen Kronprinsessan Margarets Minnesfond. The study is part of a larger project within the framework of Tvärlivs (ref. 2011-242) co-financed by The Swedish Research Council Formas, The Swedish Governmental Agency for Innovation Systems Vinnova and Fazer AB.

\section{References}

1. Cust AE, Skilton MR, van Bakel MME, Halkjaer J, Olsen A, Agnoli C, et al. Total dietary carbohydrate, sugar, starch and fiber intakes in the European Prospective Investigation into Cancer and Nutrition. Eur J Clin Nutr 2009; 63(Suppl 4): $37-60$.

2. Kyrø C, Skeie G, Dragsted LO, Christensen J, Overvad K, Hallmans G, et al. Intake of whole grain in Scandinavia: intake, sources and compliance with new national recommendations. Scand J Public Health 2012; 40: 76-84.

3. Frølich W, Åman P, Tetens I. Whole grain foods and health - a Scandinavian perspective. Food Nutr Res 2013; 57: 1-7.

4. Nordiska ministerrådet (2014). Nordic nutrition recommendations 2012: integrating nutrition and physical activity. Copenhagen: Nordic Council of Ministers.

5. Amcoff E, Edberg A, Enghardt Barbieri H, Lindroos AK, Nälsen C, Pearson M, et al. Riksmaten 2010-11, Food and nutrient intake among adults in Sweden (in Swedish) Report. Uppsala: Swedish National Food Agency; 2012.

6. Lang R, Jebb SA. Who consumes whole grains, and how much? P Nutr Soc 2003; 62: 123-7.

7. Slavin J. Whole grains and human health. Nutr Res Rev 2004; 17: 99-110.

8. Ye EQ, Chacko SA, Chou EL, Kugizaki M, Liu S. Greater whole-grain intake is associated with lower risk of type 2 diabetes, cardiovascular disease, and weight gain. J Nutr 2012; 142: $1304-13$.

9. Aune D, Chan DS, Lau R, Vieira R, Greenwood DC, Kampman E, et al. Dietary fiber, whole grains, and risk of colorectal cancer: systematic review and dose-response metaanalysis of prospective studies. BMJ 2011; 343: d6617.

10. Becker W, Busk L, Mattisson I, Sand S. Advice about whole grain 2009 - background and scientific basis (in Swedish) Report 10. Uppsala: Swedish National Food Agency; 2012.

11. Rosén L, Silva L, Andersson U, Holm C, Östman E, Björck I. Endosperm and whole grain rye breads are characterized by low post-prandial insulin response and a beneficial blood glucose profile. Nutr J 2009; 8: 42. DOI: 10.1186/1475-2891-8-42.
12. Leinonen K, Liukkonen $\mathrm{K}$, Poutanen $\mathrm{K}$, Uusitupa $\mathrm{M}$, Mykkänen H. Rye bread decreases postprandial insulin response but does not alter glucose response in healthy Finnish subjects. Eur J Clin Nutr 1999; 53: 262-7.

13. Isaksson H, Fredriksson H, Andersson R, Olsson J, Åman P. Effect of rye bread breakfasts on subjective hunger and satiety: a randomized controlled trial. Nutr J 2009; 8: 39. DOI: 10.1186/ 1475-2891-8-39.

14. Rosén LA, Östman EM, Björck IM. Effects of cereal breakfasts on postprandial glucose, appetite regulation and voluntary energy intake at a subsequent standardized lunch; focusing on rye products. Nutr J 2011; 10: 7. DOI: 10.1186/1475-2891-10-7.

15. Mann J, Nye ER. Fad diets in Sweden, of all places. Lancet 2009; 374: 767-9.

16. Gunnarsson A, Elam M. Food Fight! The Swedish LowCarb/High Fat (LCHF) movement and the turning of science popularisation against the scientists. Sci Cult 2012; 21: 315-34.

17. Johansson I, Nilsson L, Stegmayr B, Boman K, Hallmans G, Winkvist A. Associations among 25-year trends in diet, cholesterol and BMI from 140,000 observations in men and women in Northern Sweden. Nutr J 2012; 11: 40. DOI: 10.1186/14752891-11-40.

18. Becker W, Pearson M. Riksmaten 1997-98, dietary habits and nutrient intake in Sweden: analysis of results and method (in Swedish) Report. Uppsala: Swedish National Food Agency; 2002.

19. Seiluri T, Lahelma E, Rahkonen O, Lallukka T. Changes in socio-economic differences in food habits over time. Public Health Nutr 2011; 14: 1919-26.

20. Prättälä R, Helasoja V, Mykkanen H. The consumption of rye bread and white bread as dimensions of health lifestyles in Finland. Public Health Nutr 2001; 4: 813-19.

21. Worsley A. The behavioural and demographic contexts of white bread consumption. Br Food J 2003; 105: 695-9.

22. Livsmedelskonsumtion och näringsinnehåll Uppgifter t.o.m. 2012. Food consumption and nutritive values, data up to 2012. Jönköping, Sweden: Swedish Board of Agriculture; 2013.

23. Wirfält E, McTaggart A, Pala V, Gullberg B, Frasca G, Panico $\mathrm{S}$, et al. Food sources of carbohydrates in a European cohort of adults. Public Health Nutr 2002; 5: 1197-215.

24. Swedish food composition database version Riksmaten, 2010 11. Uppsala: Swedish National Food Agency.

25. Hair JF. Multivariate data analysis: a global perspective. Upper Saddle River, NJ: Pearson Education; 2010.

26. Hosmer DW, Lemeshow S. Applied logistic regression. New York: Wiley; 2000.

27. SPSS. IBM SPSS Statistics. Version 19.0.0 ed2010.

28. Berg CM, Lappas G, Strandhagen E, Wolk A, Torén K, Rosengren A, et al. Food patterns and cardiovascular disease risk factors: the Swedish INTERGENE research program. Am J Clin Nutr 2008; 88: 289-97.

29. Kyrø C, Skeie G, Dragsted LO, Christensen J, Overvad K, Hallmans $G$, et al. Intake of whole grains in Scandinavia is associated with healthy lifestyle, socio-economic and dietary factors. Public Health Nutr 2011; 14: 1787-95.

30. Hupkens CLH, Knibbe RA, Drop MJ. Social class differences in women's fat and fiber consumption: a cross-national study. Appetite 1997; 28: 131-49.

31. Arvola A, Lähteenmäki L, Dean M, Vassallo M, Winkelmann M, Claupein E, et al. Consumers' beliefs about whole and refined grain products in the UK, Italy and Finland. J Cereal Sci 2007; 46: 197-206.

32. Kantor LS, Variyam JN, Allshouse JE, Putnam JJ, Lin BH. Choose a variety of grains daily, especially whole grains: a challenge for consumers. J Nutr 2001; 131: 473S-86S. 
33. Mejborn H, Biltoft-Jensen A, Trolle E, Tetens I. Fuldkorn. Definition og vidensgrundlag for anbefaling af fuldkornsindtag $i$ Danmark (Wholegrain. Definition and scientific background for recommendations of Wholegrain intake in Denmark). Soebort: Technical University of Denmark, National Food Institute; 2008.

34. Bakke A, Vickers Z. Consumer liking of refined and whole wheat breads. J Food Sci 2007; 72: S473-80.

35. Heiniö RL, Liukkonen KH, Katina K, Myllymäki O, Poutanen $\mathrm{K}$. Milling fractionation of rye produces different sensory profiles of both flour and bread. LWT - Food Sci Tech 2003; 36: $577-83$.

36. Kihlberg I, Risvik E. Consumers of organic foods - value segments and liking of bread. Food Qual Prefer 2007; 18: 47181.

37. Nørgaard MK, Brunsø K. Family conflicts and conflict resolution regarding food choices. J Cons Behav 2011; 10: 141-51.

38. Berg C, Jonsson I, Conner M, Lissner L. Perceptions and reasons for choice of fat- and fiber-containing foods by Swedish schoolchildren. Appetite 2003; 40: 61-7.

39. Pohjanheimo T, Luomala H, Tahvonen R. Finnish adolescents' attitudes towards wholegrain bread and healthiness. J Sci Food Agric 2010; 90: 1538-44.

40. Cooke L. The importance of exposure for healthy eating in childhood: a review. J Hum Nutr Diet 2007; 20: 294-301.

41. Orfanos P, Naska A, Trichopoulou A, Grioni S, Boer JMA, van Bakel MME, et al. Eating out of home: energy, macro- and micronutrient intakes in 10 European countries. The European Prospective Investigation into Cancer and Nutrition. Eur J Clin Nutr 2009; 63(Suppl 4): S239-62.

42. Yeomans MR. The role of learning in development of food preferences. In: Shepherd R, Raats M, eds. The psychology of food choice. Wallingford, Oxfordshire, UK: CABI in association with the Nutrition Society; 2006, pp. 93-112.
43. Gibson RS. Principles of nutritional assessment. New York: Oxford University Press; 2005.

44. Binkley JK, Golub A. Consumer demand for nutrition versus taste in four major food categories. Agric Econ 2011; 42: 65-74.

45. Scazzina F, Siebenhandl-Ehn S, Pellegrini N. The effect of dietary fiber on reducing the glycaemic index of bread. Br J Nutr 2013; 109: 1163-74.

46. Morton LM, Cahill J, Hartge P. Reporting participation in epidemiologic studies: a survey of practice. Am $\mathrm{J}$ Epidemiol 2006; 163: 197-203.

47. Tolonen H, Helakorpi S, Talala K, Helasoja V, Martelin T, Prättälä R. 25-Year trends and socio-demographic differences in response rates: Finnish adult health behaviour survey. Eur J Epidemiol 2006; 21: 409-15.

48. Norkost 3 (2012). En landsomfattende kostholdsundersøkelse blant menn og kvinner i Norge i alderen 18-70 år, 2010-11, A nation-wide dietary survey among Norwegian men and women aged 18-70 years, 2010-11 (in Norwegian) Report. Oslo: Helsodirektoratet.

49. Korkeila K, Suominen S, Ahvenainen J, Ojanlatva A, Rautava $\mathrm{P}$, Helenius $\mathrm{H}$, et al. Non-response and related factors in a nation-wide health survey. Eur J Epidemiol 2001; 17: 991-9.

50. Simunaniemi AM, Andersson A, Nydahl M. Fruit and vegetable consumption close to recommendations. A partly webbased nationwide dietary survey in Swedish adults. Food Nutr Res 2009;53. DOI: 10.3402/fnr.v53i0.2023.

\footnotetext{
*Pernilla Sandvik

Department of Food, Nutrition and Dietetics

Uppsala University

SE-75I 05 Uppsala, Sweden

Email: pernilla.sandvik@ikv.uu.se
} 